\title{
Incidental lesion in the femoral metaphysis
}

\section{Vibhor Wadhwa • Kyung Jin Suh • Jae Hyuck Yi • Avneesh Chhabra}

Published online: 20 April 2013

(C) ISS 2013

A 46 year old man was incidentally found to have a lytic lesion in the right femoral metaphysis (Figs. 1, 2, 3, 4 and 5).

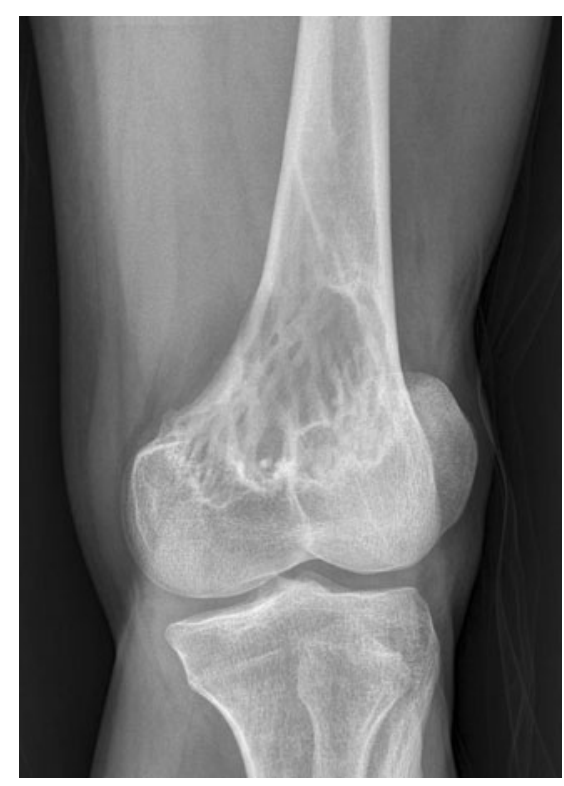

Fig. 1 AP radiograph of the right knee

The diagnosis can be found at doi: 10.1007/s00256-013-1623-6.

V. Wadhwa $\cdot$ A. Chhabra $(\triangle)$

The Russell H. Morgan Department of Radiology and Radiological Science, Johns Hopkins University School of Medicine, 601, N. Caroline Street,

Baltimore, MD 21287, USA

e-mail: achhabr6@jhmi.edu

V. Wadhwa

e-mail: vibhorwadhwa90@gmail.com

\section{K. J. Suh}

Department of Radiology, Gyungju Hospital College of Medicine, Dongguk University, Gyungju-Si, Republic of Korea e-mail: Kyungjin.suh@gmail.com

\section{J. H. Yi}

Department of Radiology, Kyungpook National University Hospital, Daegu, Republic of Korea

e-mail: yijh@knu.ac.kr

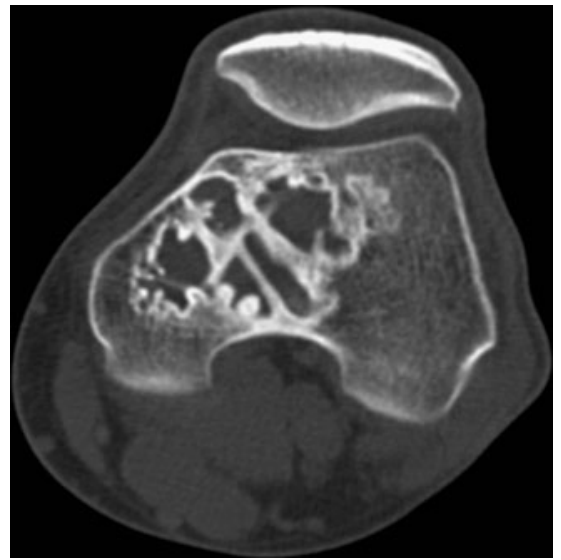

Fig. 2 Axial CT image of the right knee

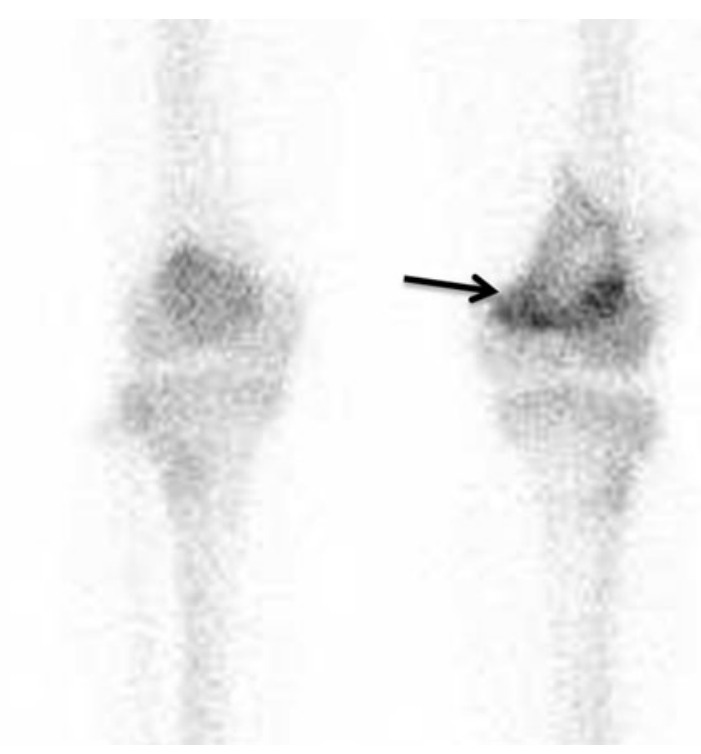

Fig. 3 Anterior planar delayed imaging of both knees from Tc $99 \mathrm{~m}$ MDP bone scan 
Fig. 4 Coronal T1W (a), fat suppressed $\mathrm{T} 2 \mathrm{~W}(\mathbf{b})$ and post intravenous contrast fat suppressed T1W (c) images of the right knee with distal femur
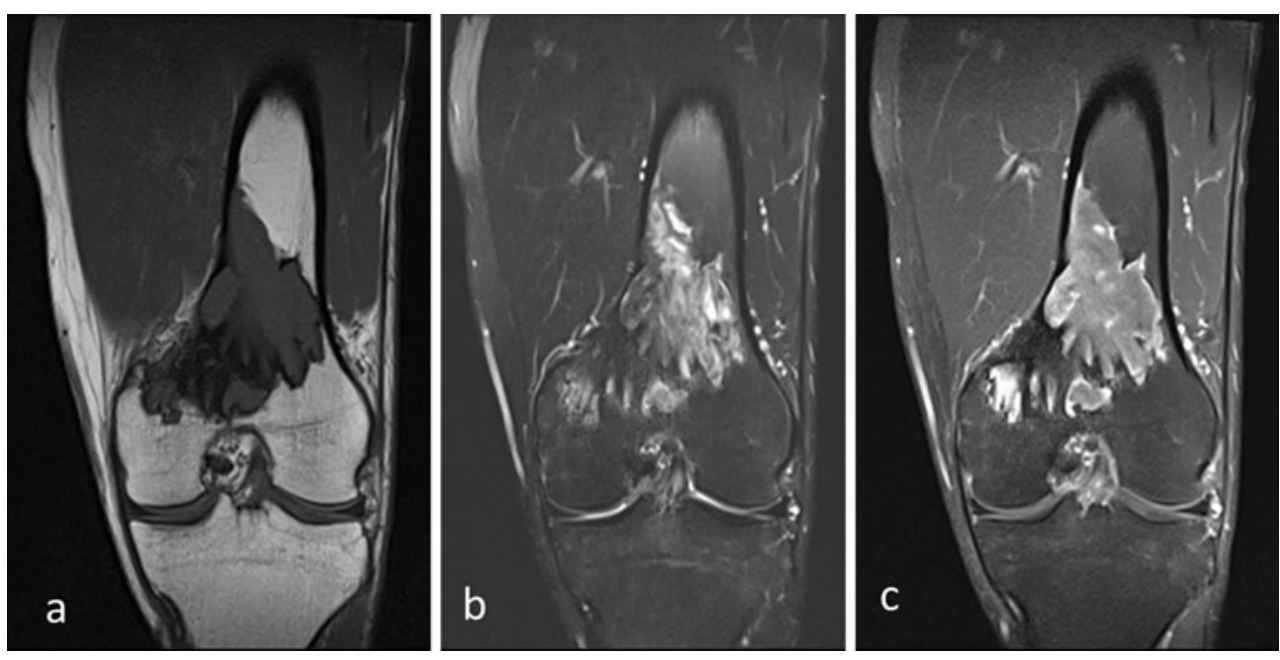

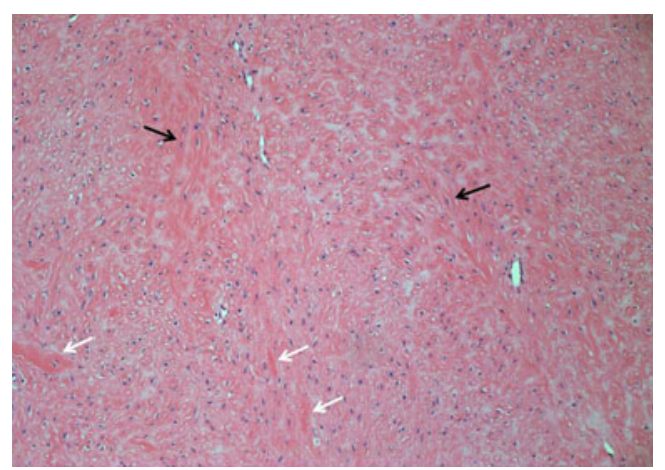

Fig. 5 Histopathology image. (Hematoxylin-eosin stain; Magnification $\times 100$ )

Disclosures Dr. Avneesh Chhabra has research grants from GE-AUR (GERRAF), Siemens Medical Solutions and Integra Life Sciences. He also serves as a research consultant with Siemens CAD group. 